\title{
Prognostic Implication of 15-Hydroxyprostaglandin Dehydrogenase Down-Regulation in Patients with Colorectal Cancer
}

\author{
Pil Sung Kang, Jin Ha Kim, Ok In Moon, Sung Chul Lim', Kyung Jong Kim \\ Departments of Surgery and ${ }^{1}$ Pathology, Chosun University School of Medicine, Gwangju, Korea
}

Purpose: Prostaglandin (PG) $\mathrm{E}_{2}$ is known to be closely related to cancer progression and is inactivated by 15-hydroxyprostaglandin dehydrogenase (PGDH). 15-PGDH is shown to have tumor suppressor activity and to be down-regulated in various cancers, including colorectal cancer (CRC). Therefore, we evaluated the expression of 15-PGDH and its prognostic effect in patients with CRC.

Methods: 15-PGDH expression was examined by using immunohistochemistry in 77 patients with CRC. Its prognostic significance was statistically evaluated.

Results: Negative 15-PGDH expression was noted in 55.8\% of the 77 cases of CRC. 15-PGDH expression showed no correlation with any of the various clinicopathologic parameters. The status of lymph node metastasis, tumor-node-metastasis stages, and pre-operative carcinoembryonic antigen levels showed significant prognostic effect. However, univariate analysis revealed down-regulation of $15-\mathrm{PGDH}$ not to be a predictor of poor survival. The 5-year overall survival rate was $71.7 \%$ in the group with positive expression of $15-\mathrm{PGDH}$ and $67.1 \%$ in the group with negative expression of 15-PGDH, but this difference was not statistically significant $(\mathrm{P}=0.751)$.

Conclusion: 15-PGDH was down-regulated in $55.8 \%$ of the colorectal cancer patients. However, down-regulation of $15-$ PGDH showed no prognostic value in patients with CRC. Further larger scale or prospective studies are needed to clarify the prognostic effect of 15-PGDH down-regulation in patients with colorectal cancer.

Keywords: Colorectal neoplasms; 15-hydroxyprostaglandin dehydrogenase; Down-regulation; Immunohistochemistry; Prognosis

\section{INTRODUCTION}

The adenoma-carcinoma sequence and its related genes was reported in the carcinogenesis of colorectal cancer in the 1990s [1]. However, more molecular biological mechanisms have been clarified in recent years. Several pathways are known to be involved in

Received: April 30, 2012 - Accepted: September 14, 2012

Correspondence to: Kyung Jong Kim, M.D.

Department of Surgery, Chosun University Hospital, 365 Pilmun-daero,

Dong-gu, Gwangju 501-717, Korea

Tel: +82-62-220-3068, Fax: +82-62-228-3441

Email: kjkim@chosun.ac.kr

The main idea of this research was presented orally at the Korean Society of Coloproctology in 2011.

(c) 2012 The Korean Society of Coloproctology

This is an open-access article distributed under the terms of the Creative Commons Attribution NonCommercial License (http://creativecommons.org/licenses/by-nc/3.0) which permits unrestricted noncommercial use, distribution, and reproduction in any medium, provided the original work is properly cited. the process of colorectal carcinogenesis, including the chromosomal instability pathway, the $\mathrm{CpG}$ island methylator phenotype pathway, the microsatellite instability pathway, and the serrated pathway [2]. In addition, chronic inflammations may play a crucial role in cancer initiation. As proof, the incidence of colorectal cancer has been shown to be greater in patients with inflammatory bowel disease [3]. Prostaglandin (PG) is identified as a substance that is profoundly related to inflammatory response. PGs are bioactive lipids produced from arachidonic acid converted from the metabolism of phospholipids in membranes, including $\mathrm{PGE}_{2}$, $\mathrm{PGD}_{2}, \mathrm{PGF}_{2} \alpha, \mathrm{PGI}_{2}$, and thromboxane $\mathrm{A}_{2}$. They play crucial roles in physiological aspects such as renal function maintenance, gastrointestinal function maintenance, and the regulation of vascular homeostasis, as well as, during the inflammatory response [4].

Among the different $\mathrm{PGs}, \mathrm{PGE}_{2}$ is especially known to promote carcinogenesis and cancer progression by activating cancer cells, promoting angiogenesis, and inhibiting apoptosis [5]. Cyclooxygenase (COX) plays the most significant role in $\mathrm{PGE}_{2}$ production. 
There are mainly two types of COX. COX-1 is involved in normal physiological function by maintaining a constant amount while COX-2 is expressed in an increased amount induced by certain stimulation. Inflammatory response is known to be involved in the formation of $\mathrm{PGE}_{2}$ [6]. Moreover, COX-2 is known to play a role as an oncogene, and COX-2 is over-expressed in various solid tumors, including colorectal cancer. Pharmacological inhibitors of COX, such as aspirin, sulindac and celecoxib, have been demonstrated to lower the incidence of colon cancer, gastric cancer, and esophageal cancer, and the use of these inhibitors has been shown through epidermiologic studies to lower the mortality rates of these cancers [7]. However, the long-term use of COX-2 suppressors is known to have serious adverse effects on the gastrointestinal and cardiovascular systems. The causes are thought to be that $\mathrm{COX}-2$ suppressors not only inhibit $\mathrm{PGE}_{2}$ production but also inhibit the formation of other various PGs at the same time [8]. Therefore, studies on substances that can selectively reduce $\mathrm{PGE}_{2}$ only are essential. Microsomal prostaglandin E synthase is currently known to overcome the limitations of COX-2 and to involve the synthesis of $\mathrm{PGE}_{2}$ selectively. Furthermore, 15-hydroxyprostaglandin dehydrogenase (15-PGDH) is known as enzymedegrading 15-PGDH [9]. Because these enzymes are involved in the metabolism of $\mathrm{PGE}_{2}$ by working at the lower phase of COX-2, they are anticipated to regulate $\mathrm{PGE}_{2}$ selectively and avoiding the adverse effects of COX-2.

15-PGDH is an enzyme that converts $\mathrm{PGE}_{2}$ into 15-keto-prostaglandin by working on 15(S)-hydroxyl groups, eventually leading to inactivation of $\mathrm{PGE}_{2}$. Recent studies have demonstrated that decreased 15-PGDH has a profound relationship with carcinogenesis and cancer progression. Decreased 15-PGDH expression was observed in patients with colorectal cancer, breast cancer, prostate cancer, lung cancer, thyroid cancer, gastric cancer and other cancers [10, 11]. Moreover, Myung et al. [12] reported an increase in colon tumorigenesis in 15-PGDH knock-out mice and proposed that 15-PGDH played the role of a tumor suppressor. The authors of this study reported that the expression of 15-PGDH was lower in colorectal cancer and that it was inversely correlated with the expression of vascular endothelial growth factor (VEGF), which is closely correlated with angiogenesis [13]. Such a result suggested a significant relationship between down-regulation of 15-PGDH and cancer progression. As the role of $15-\mathrm{PGDH}$ as a tumor suppressor has been proven, a hypothesis that down-regulation of 15PGDH will work as a poor prognostic parameter can be formulated. However, few studies have been carried out on the role of $15-\mathrm{PGDH}$ as a prognostic predictor for colorectal cancer. Therefore, this study aims to investigate the role of 15-PGDH expression in colorectal cancer patients as a prognostic parameter.

\section{METHODS}

\section{Patients and tissues}

Seventy-seven patients who had undergone surgical resection for a primary sporadic colorectal carcinoma by a single colorectal surgeon at the Department of Surgery, Chosun University Hospital, between March 2002 and December 2005 were included in this study. The study excluded patients who regularly took aspirin or other anti-inflammatory drugs, those who underwent preoperative chemotherapy or radiotherapy, and those who had evidence of hereditary or familial CRC syndrome. Enrolled patients were followed up until death or December 2008. The mean follow-up period was 52.5 months (4 to 93 months), and the mean age of the patients was $63.9( \pm 11.4)$ years old at the time of surgery. Surgically-resected tissues were classified by pathologists according to their characteristics, including histological differentiation, the depth of tumor invasion, lymph node invasion, the presence of distant metastasis, etc. In all cases, archived hematoxylin and eosin-stained tissue slides were obtained for confirmation of pathological features and for the selection of suitable tissue blocks for immunohistochemical analysis.

\section{Immunohistochemical staining}

The expression of 15-PGDH was observed in resected colorectal
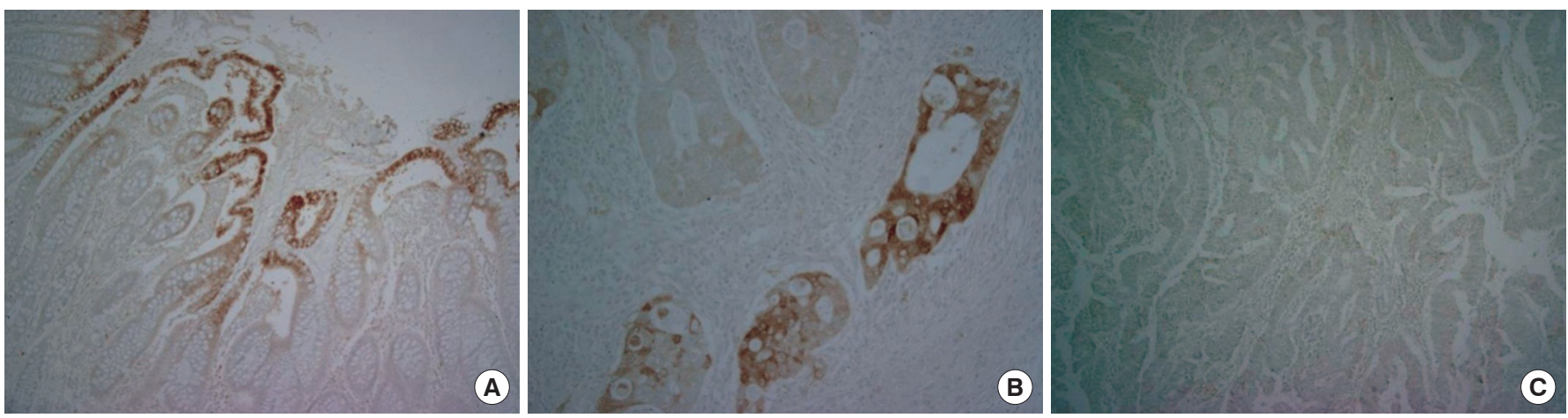

Fig. 1. Immunohistochemistry for 15-prostaglandin dehydrogenase (15-PGDH) in normal colon tissues and in colorectal cancer tissues: (A) normal 15-PGDH expression in colon mucosa $(\times 100)$, (B) positive 15-PGDH expression in cancer tissue $(\times 200)$, and $(\mathrm{C})$ negative $15-\mathrm{PGDH}$ expression in cancer tissue $(\times 100)$. 
cancer tissue by immunohistochemical staining. Paraffin blocks were cut into 4-micrometer-thick pieces and washed after the paraffin had been removed with xylene and alcohol. Subsequently, sections were immersed in sodium citrate buffer ( $\mathrm{pH}$ 6.0) and autoclaved at $121^{\circ} \mathrm{C}$ for 15 minutes for antigen retrieval. In addition, they were treated in methanol with $3 \%$ hydrogen peroxide for 20 minutes in order to block the activation of endogenous peroxidase. Slides were treated with Ultra V Block (UltraVision Plus Detection System, Thermo Fisher Scientific Inc., Fremont, CA, USA) solution at room temperature for five minutes to inhibit non-specific binding. Rabbit polyclonal antibody (1:2,000, Novus Biologicals, Littleton, CO, USA) was used as the 15-PGDH primary antibody and was incubated at $4^{\circ} \mathrm{C}$ overnight. Each slide was washed with phosphate buffered saline (PBS) four times and incubated with biotinylated goat anti-rabbit antibody (UltraVision Plus Detection System) at room temperature for five minutes. Afterward, each slide was washed again with PBS four times and incubated with streptavidin-alkaline phosphate conjugate (UltraVision Plus Detection System). Moreover, each slide was washed four times repeatedly with PBS and developed with Fast Red tablets on a naphthol phosphate substrate. Slides were counterstained with Mayer's hematoxylin solution for 20 seconds and air-dried and enclosed with a cover slip. Normal rabbit serum immunoglobulin G (Vector Laboratories Inc., Burlingame, CA, USA) was taken as a negative control, replacing the primary antibody. The mucosa of a normal colon was used as an internal control. All our experiments were performed in duplicate.

\section{Determination of immunohistochemical staining}

Each slide was assessed by pathologists who were unaware of the patients' clinical information. A patient was defined as being positive when 15-PGDH was expressed in more than $30 \%$ of the cancer cells of each tissue sample. In contrast, a patient was defined as being negative or down-regulated when 15-PGDH was expressed in less than $30 \%$ of those cells [14].

\section{Statistical analysis}

The relationship between 15-PGDH expression and clinical manifestation was analyzed using a chi-square test and Fisher's exact test. Patients' survival rates were analyzed using the Kaplan-Meier method. The differences in survival rates were examined using a log-rank test. P-values of less than 0.05 were defined as statistically significant. SPSS ver. 17.0 (SPSS Inc., Chicago, IL, USA) was used for data analysis.

\section{RESULTS}

The expression of 15-PGDH was observed in normal mucosa of all colorectal cancer patients. Expression of 15-PGDH was downregulated in 34 (55.8\%) out of 77 patients (Fig. 1). The expression rates of $15-\mathrm{PGDH}$ were $50 \%$ and $40 \%$ in colon and rectal cancers, respectively. However, no statistical difference was found between the two groups. Down-regulation of 15-PGDH was revealed in the patient group with increased preoperative carcinoembryonic antigen (CEA) levels, indicating a statistically marginal significance $(P=0.056)$. No differences were detected in 15-PGDH expression depending on the depth of tumor invasion (T-stage), lymph node invasion (N-stage), the presence of distant metastasis (M-stage), etc (Table 1).

The study analyzed various clinicopathologic factors affecting the survival rate of patients with colorectal cancer. Long-term survival was anticipated in the groups with no lymph node metastasis, low tumor-node-metastasis (TNM) stages, and normal preop-

Table 1. Correlation of 15-prostaglandin dehydrogenase (15-PGDH) expression with clinicopathologic parameters

\begin{tabular}{|c|c|c|}
\hline Parameter & 15-PGDH (\%) & P-value \\
\hline All cases & $34 / 77$ (44.2) & \\
\hline Sex & & 0.295 \\
\hline Male & 20/39 (51.3) & \\
\hline Female & 14/38 (36.8) & \\
\hline Age (yr) & & 0.655 \\
\hline$<60$ & $12 / 24(50.0)$ & \\
\hline$\geq 60$ & 22/53 (41.5) & \\
\hline Site & & 0.523 \\
\hline Colon & 16/32 (50.0) & \\
\hline Rectum & $18 / 45(40.0)$ & \\
\hline CEA level & & 0.056 \\
\hline Normal $^{\mathrm{a}}$ & $18 / 40(45.0)$ & \\
\hline Elevated & 7/22 (31.8) & \\
\hline Depth of invasion & & 0.773 \\
\hline $\mathrm{T} 1$ & $3 / 5(60.0)$ & \\
\hline $\mathrm{T} 2$ & $7 / 17$ (41.2) & \\
\hline T3 & 22/51 (43.1) & \\
\hline T4 & 2/4 (50.0) & \\
\hline Lymph node metastasis & & 0.513 \\
\hline Negative & 19/44 (43.2) & \\
\hline Positive & 15/33 (45.5) & \\
\hline TNM stage & & 0.742 \\
\hline I & 8/18 (44.4) & \\
\hline$\|$ & 11/24 (45.8) & \\
\hline III & 13/30 (43.3) & \\
\hline IV & $2 / 5(40.0)$ & \\
\hline Differentiation & & 0.546 \\
\hline Well & 9/16 (56.3) & \\
\hline Moderate/poor & 22/54 (40.7) & \\
\hline
\end{tabular}

CEA, carcinoembryonic antigen; TNM, tumor-node-metastasis.

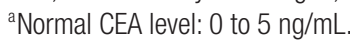


Table 2. Predictive factors for long-term survival of colorectal cancer patients (univariate analysis)

\begin{tabular}{|c|c|c|c|}
\hline Variable & $\begin{array}{c}\text { No. of } \\
\text { patients }\end{array}$ & Relative risk (95\% Cl) & P-value \\
\hline \multicolumn{4}{|c|}{ Depth of invasion } \\
\hline $\mathrm{T} 1$ & 5 & 1 & \\
\hline T2 & 17 & $0.31(0.02-4.91)$ & 0.404 \\
\hline T3 & 51 & 2.18 (0.29-16.27) & 0.448 \\
\hline T4 & 4 & 7.85 (0.81-76.15) & 0.075 \\
\hline \multicolumn{4}{|c|}{ Lymph node metastasis } \\
\hline Negative & 44 & 1 & 0.039 \\
\hline Positive & 33 & $2.39(1.05-5.46)$ & \\
\hline \multicolumn{4}{|l|}{ TNM stage } \\
\hline I & 18 & 1 & \\
\hline$\|$ & 24 & $1.94(0.38-10.01)$ & 0.43 \\
\hline III & 30 & $4.02(0.90-17.96)$ & 0.069 \\
\hline IV & 5 & 19.91 (3.79-104.69) & $<0.001$ \\
\hline \multicolumn{4}{|l|}{ CEA level } \\
\hline Normal $^{\mathrm{a}}$ & 40 & 1 & \\
\hline Elevated & 22 & 2.518 (1.07-5.96) & 0.036 \\
\hline \multicolumn{4}{|c|}{ Expression of 15-PGDH } \\
\hline Negative & 43 & 1 & \\
\hline Positive & 34 & 0.88 (0.39-1.98) & 0.753 \\
\hline
\end{tabular}

$\mathrm{Cl}$, confidence interval; TNM, tumor-node-metastasis; CEA, carcinoembryonic antigen; 15-PGDH, 15-prostaglandin dehydrogenase.

aNormal CEA level: 0 to $5 \mathrm{ng} / \mathrm{mL}$.

erative CEA levels (Table 2). When the survival analysis was performed using a Kaplan-Meier analysis, the 5-year survival rates were $88.9 \%$ at stage I, $79.2 \%$ at stage II, $57.9 \%$ at stage III and $20 \%$ at stage IV, showing statistical significance $(\mathrm{P}<0.001)$. Moreover, significant differences were observed in the disease-free survival rate depending on $\mathrm{TNM}$ stage $(\mathrm{P}=0.033$, data not shown).

The five-year survival rates were $71.7 \%$ in patients with 15 PGDH expression and $67.1 \%$ in patients with down-regulation of 15-PGDH, but this difference was not statistically significant (Fig. 2). The difference in survival rates of COX-2-positive patients were analyzed depending on the status of 15-PGDH expression because 15-PGDH, as well as COX-2, plays a significant role in relation to $\mathrm{PGE}_{2}$ metabolism. The five-year survival rates of COX-2 positive patients were $73.3 \%$ in 15-PGDH-positve patients and $68.9 \%$ in 15-PGDH-negative patients, but this difference was not statistically significant (data not shown).

\section{DISCUSSION}

The authors of the study intended to investigate the prognostic implication of 15-PGDH, which is known for its role as a tumor

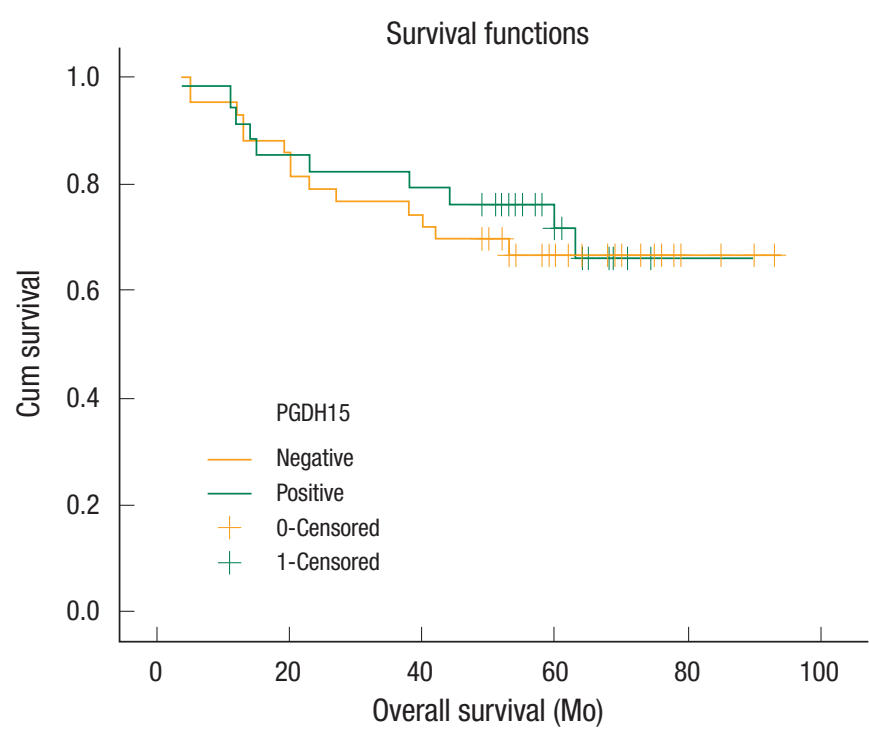

Fig. 2. Overall survival according to the 15-prostaglandin dehydrogenase (15-PGDH) expression as obtained by using a Kaplan-Meier analysis $(\mathrm{P}=0.752)$.

suppressor gene, for patients with colorectal cancer. However, statistical significance was not detected. $\mathrm{NAD}(+)$-dependent 15-PGDH with a molecular weight of $29 \mathrm{kDa}$ is involved in $\mathrm{PGE}_{2}$ metabolism, and it is known to be activated as a homodimer [15].

Yan et al. [16] verified that 15-PGDH mRNA and protein were both highly expressed at the normal colon mucosa while almost no expression was assessed in colon cancer tissues and that xenograft tumor formation of colon cancer cells was lower in nude mice when 15-PGDH was transfected. Thus, they suggested the effects of 15-PGDH expression as a tumor suppressor in colon cancer. During a similar period, Backlund et al. [17] reported that the activity and the expression of 15-PGDH was lower in colon cancer cells, breast cancer cells, lung cancer cells and the adenomas in Apc Min mouse and that they were higher in normal colon tissues of humans or mice. Myung et al. [12] examined the role of 15-PGDH as a tumor suppressor gene in vivo. Moreover, 15-PGDH gene knockout in an Apc Min mouse model increased the incidence of tumors in the mouse colon by a factor of 7.6. They suggested that $15-\mathrm{PGDH}$ played the role of a tumor suppressor by using an in vivo experiment in which C57BL/6J rats were administered the carcinogen azoxymethane to demonstrate an increased incidence of adenomas or intraepithelial carcinomas in 15-PGDH gene knockout mice. In previous studies, we also found that 15-PGDH was down-regulated in the tissues of colorectal cancer patients and that the expressions of 15-PGDH and VEGF were inversely correlated, suggesting a tumor progression effect of 15-PGDH downregulation in colorectal cancer [13]. Besides colorectal cancer, 15PGDH is known for its role as a tumor suppressor gene in various other cancers. The expression of 15-PGDH is markedly lower in lung cancer tissues compared to adjacent normal pulmonary tis- 
sues. In conjunction with up-regulation of COX-2 and PGE synthase, down-regulation of 15 -PGDH increased $\mathrm{PGE}^{2}$ levels, consequently causing proliferation of a tumor [18]. Down-regulation of 15 -PGDH was detected in around $40 \%$ of breast cancer tissues and is reported to be correlated with the expression of the estrogen receptor. When 15-PGDH was transfected in breast cancer cells, the tumor formation rate was lower in rats [19]. According to the expression of 15-PGDH in gastric cancer, 15-PGDH mRNA was five times lower in cancer tissues compared to adjacent normal tissues. $15-\mathrm{PGDH}$ protein was down-regulated in $65 \%$ of the patients. Wolf et al. [19] also reported that the mechanism of 15PGDH down-regulation was methylation in the promoter region of 15-PGDH [20]. Kaliberova et al. [21] attempted gene therapy on nude mice by transfecting 15-PGDH to breast cancer cells (2LMP) and colon cancer cells (LS174T). The gene therapy of 15PGDH delayed the tumor growth in both breast cancer cells and colon cancer cells. When combined 15-PGDH gene therapy and bevacizumab were concurrently applied to LS174T cells, tumor growth declined significantly compared to the sole use of bevacizumab.

Although the tumor suppressor activity of 15-PGDH has already been verified by many studies, only a few studies have investigated the role of 15-PGDH for its prognostic implications [21-23]. Tatsuwaki et al. [22] reported that decreased expression of 15-PGDH was observed in 35 out of 71 gastric cancer patients and that it was an independent adverse prognostic parameter. On the other hand, Thiel et al. [20] reported that 15-PGDH expression status in gastric cancer tissues had no significant relationship with prognostic implication. Lehtinen et al. [23] paradoxically reported that high expression of 15-PGDH mRNA was correlated with poor prognosis in 295 patients with breast cancer. Although the present study identified that the five-year survival rate was slightly higher when 15-PGDH was expressed, no statistical difference was detected when 15-PGDH expression was used as a prognostic parameter.

The authors think this study is worthy of note as the first research to investigate the role of 15-PGDH as a prognostic parameter in colorectal cancer. Prospective studies with a larger number of study subjects are thought to be essential because the results on the role of 15-PGDH as a prognostic parameter differ extensively from study to study and are often contradictory.

In conclusion, in this study, 15-PGDH was down-regulated in $55.8 \%$ of the patients with colorectal cancer. No significant correlation was detected between the expression of 15-PGDH and the prognostic implications for patients with colorectal cancer. However, further studies with larger study populations are thought to be crucial because this study was limited to a small number of subjects.

\section{CONFLICT OF INTEREST}

No potential conflict of interest relevant to this article was reported.

\section{ACKNOWLEDGMENTS}

This research was supported by a grant from Chosun University in 2008 .

\section{REFERENCES}

1. Fearon ER, Vogelstein B. A genetic model for colorectal tumorigenesis. Cell 1990;61:759-67.

2. Worthley DL, Leggett BA. Colorectal cancer: molecular features and clinical opportunities. Clin Biochem Rev 2010;31:31-8.

3. Harpaz N, Polydorides AD. Colorectal dysplasia in chronic inflammatory bowel disease: pathology, clinical implications, and pathogenesis. Arch Pathol Lab Med 2010;134:876-95.

4. Narumiya S, Sugimoto Y, Ushikubi F. Prostanoid receptors: structures, properties, and functions. Physiol Rev 1999;79:1193-226.

5. Wang D, Dubois RN. Prostaglandins and cancer. Gut 2006;55: 115-22.

6. Williams CS, Mann M, DuBois RN. The role of cyclooxygenases in inflammation, cancer, and development. Oncogene 1999;18: 7908-16.

7. Thun MJ, Namboodiri MM, Heath CW Jr. Aspirin use and reduced risk of fatal colon cancer. N Engl J Med 1991;325:1593-6.

8. Kerr DJ, Dunn JA, Langman MJ, Smith JL, Midgley RS, Stanley A, et al. Rofecoxib and cardiovascular adverse events in adjuvant treatment of colorectal cancer. N Engl J Med 2007;357:360-9.

9. Nakanishi M, Montrose DC, Clark P, Nambiar PR, Belinsky GS, Claffey KP, et al. Genetic deletion of mPGES-1 suppresses intestinal tumorigenesis. Cancer Res 2008;68:3251-9.

10. Tai HH, Tong M, Ding Y. 15-hydroxyprostaglandin dehydrogenase (15-PGDH) and lung cancer. Prostaglandins Other Lipid Mediat 2007;83:203-8.

11. Myung SJ, Kim IH. Role of prostaglandins in colon cancer. Korean J Gastroenterol 2008;51:274-9.

12. Myung SJ, Rerko RM, Yan M, Platzer P, Guda K, Dotson A, et al. 15-Hydroxyprostaglandin dehydrogenase is an in vivo suppressor of colon tumorigenesis. Proc Natl Acad Sci U S A 2006;103:12098102.

13. Lim SC, Cho H, Lee TB, Choi CH, Min YD, Kim SS, et al. Impacts of cytosolic phospholipase A2, 15-prostaglandin dehydrogenase, and cyclooxygenase-2 expressions on tumor progression in colorectal cancer. Yonsei Med J 2010;51:692-9.

14. Yoo NJ, Jeong EG, Lee SH, Lee SH. Expression of 15-hydroxyprostaglandin dehydrogenase, a COX-2 antagonist and tumour suppressor, is not altered in gastric carcinomas. Pathology 2007; 39:174-5.

15. Ensor CM, Yang JY, Okita RT, Tai HH. Cloning and sequence analysis of the cDNA for human placental $\mathrm{NAD}(+)$-dependent 15-hydroxyprostaglandin dehydrogenase. J Biol Chem 1990;265: 14888-91.

16. Yan M, Rerko RM, Platzer P, Dawson D, Willis J, Tong M, et al. 15-Hydroxyprostaglandin dehydrogenase, a COX-2 oncogene 


\section{Coloproctology Pil sung Kang, et al.}

antagonist, is a TGF-beta-induced suppressor of human gastrointestinal cancers. Proc Natl Acad Sci U S A 2004;101:17468-73.

17. Backlund MG, Mann JR, Holla VR, Buchanan FG, Tai HH, Musiek ES, et al. 15-Hydroxyprostaglandin dehydrogenase is downregulated in colorectal cancer. J Biol Chem 2005;280:3217-23.

18. Ding Y, Tong M, Liu S, Moscow JA, Tai HH. NAD+-linked 15-hydroxyprostaglandin dehydrogenase (15-PGDH) behaves as a tumor suppressor in lung cancer. Carcinogenesis 2005;26:65-72.

19. Wolf I, O'Kelly J, Rubinek T, Tong M, Nguyen A, Lin BT, et al. 15-hydroxyprostaglandin dehydrogenase is a tumor suppressor of human breast cancer. Cancer Res 2006;66:7818-23.

20. Thiel A, Ganesan A, Mrena J, Junnila S, Nykanen A, Hemmes A, et al. 15-hydroxyprostaglandin dehydrogenase is down-regulated in gastric cancer. Clin Cancer Res 2009;15:4572-80.
21. Kaliberova LN, Kusmartsev SA, Krendelchtchikova V, Stockard CR, Grizzle WE, Buchsbaum DJ, et al. Experimental cancer therapy using restoration of NAD+ -linked 15-hydroxyprostaglandin dehydrogenase expression. Mol Cancer Ther 2009;8:3130-9.

22. Tatsuwaki H, Tanigawa T, Watanabe T, Machida H, Okazaki H, Yamagami H, et al. Reduction of 15-hydroxyprostaglandin dehydrogenase expression is an independent predictor of poor survival associated with enhanced cell proliferation in gastric adenocarcinoma. Cancer Sci 2010;101:550-8.

23. Lehtinen L, Vainio P, Wikman H, Reemts J, Hilvo M, Issa R, et al. 15-Hydroxyprostaglandin dehydrogenase associates with poor prognosis in breast cancer, induces epithelial-mesenchymal transition, and promotes cell migration in cultured breast cancer cells. J Pathol 2012;226:674-86. 